\title{
Learning technology in Scottish higher education - a survey of the views of senior managers, academic staff and 'experts'
}

\author{
Jeff Haywood, Charles Anderson, Helen Coyle, Kate Day, Denise Haywood and \\ Hamish Macleod \\ Department of Higher and Further Education, University of Edinburgh \\ email:jeff.haywood@ed.ac.uk
}

As part of an evaluation of the Scottish Leärning Technology Dissemination Initiative (LTDI), a survey was conducted of the views of academic staff, members of computerassisted learning and staff development units, and senior managers in all Scottish higher education institutions (HEIs). Most respondents across all subject areas and types of institutions (including those who rated themselves as less experienced with use of C\&IT in teaching than their colleagues) believed that learning technology (LT) had moderate to very high potential for improving the way in which students learn. Awareness of the various agencies which have been established to promote its use in HEIs was very high, with few staff being unaware of any of them. Senior staff largely agreed that the value of these approaches lay in the improvement or maintenance of quality rather than in creating efficiency gains. Whilst there was a mostly positive view of the value of learning technology there are still significant barriers to its uptake by staff, the most important being lack of time, infrastructure, software and training, plus a failure (perceived or actual) of institutions to value teaching. The rather pessimistic view of 'experts' of the willingness of their less committed colleagues to make use of learning technology contrasted with the generally positive responses obtained from a broad group of 1,000 academic staff on their awareness of and attitudes to it. An analysis of the SHEFC's Teaching Quality Assessment reports during 1992-6 revealed substantial variability between and within subject assessments as to whether specific comments were made about IT provision and its use in learning and teaching. 


\section{Introduction}

Central concerns within the field of learning technology in higher education have been the promotion of institutional change and staff awareness and development. This focus on the need to bring about a 'culture shift' and the importance of 'change agents' is reflected in the Dearing Report (DfEE, 1997) and in Funding Council initiatives such as TLTP and TLTSN (Davies, 1995). It is common for many of us who work in this area to feel that although we see clearly the task ahead, we have little at our disposal by way of evidence about how far we have come. Much of the evidence which does exist, and which has been incorporated into lectures and reports, is anecdotal, local and small scale, although there have been some larger studies, notably the Information Technology Assisted Teaching and Learning project (ITATL, 1997), and a 1999 study of C\&IT materials funded by the Funding Councils (HEFCE, 1999a), and in the United States the national survey of desktop computing and IT in higher education (Green, 1989-99). These showed a rapidly increasing use of learning technology in higher education, and some of the limitations and restrictions which staff feel, such as technical support. However, there had been no indepth study of the subject and institution-specific influences on academic staff use of, and attitudes to, learning technology.

In August 1996 we successfully tendered for the evaluation of the first two years of the Learning Technology Dissemination Initiative (LTDI), which was a project funded by the Scottish Higher Education Funding Council to promote the use of learning technology and computer-based learning materials in SHEFC-funded Higher Education Institutions. LTDI operated from Heriot-Watt University from 1994 and in the words of the SHEFC Circular $8 / 92$, 'The aim of the programme is to make teaching and learning more productive and efficient by harnessing modern technology' (SHEFC 1992). We were commissioned to assess its impact on staff and students in Scottish higher education, and to make recommendations for future funding of learning technology by SHEFC.

Our strategy was to examine the activities and outcomes of LTDI, within the context of the views and opinions of three main groups: senior managers of HEIs, a representative sample of academic staff and 'learning technology experts' from a wide range of Scottish institutions. We needed to investigate what they thought about LTDI specifically, but to make sense of the data we also needed to know what they thought about learning technology in general and, especially for academic staff, their length of experience in higher education, their awareness of other agencies, etc:

The data presented here relate to the general views on the use of C\&IT in learning and teaching which we obtained. Their views on the value or effectiveness of LTDI, which were generally positive and in favour of some continuing SHEFC support for learning technology in Scotland, appear in our full report (which can be obtained at http://www.flp.ed.ac.uk/LTRG/LTDI.html).

\section{Survey methods}

The data were all collected over a five-month period (October 1996-February 1997). There were three main data sources: paper questionnaire, face-to-face interviews and documentary sources. 
An anonymous questionnaire survey of academic staff in all departments of all Scottish HEIs was conducted. The questionnaire, which sought information on general learning technology issues as well as those directly related to LTDI, was designed to be suitable for all academic staff, ranging from those with little or no interest or involvement in learning technology through to those who considered themselves to be 'expert'. Respondents simply worked through the questionnaire and exited at the point relevant to their level of experience with respect to LTDI. A PDF version of the survey is available at http://www.flp.ed.ac.uk/LTRG/LTDI.html.

In order to conduct the survey, a database was compiled of all academic departments in SHEFC-funded institutions. Copies of the questionnaire were then mailed to each head of department, asking them to distribute a number of questionnaires (equal to at least 20 per cent of academic staff in the department), half to staff who were noticeably interested or involved with learning technology and half to those with little or no apparent interest or involvement. In this way 'matched pairs' of academic staff across the sector were targeted, with the potential to reach around 20 per cent of the approximately 8,000 full-time academic staff (calculated from 1994-5 data in SHEFC, 1996)). At the end of January 1997, 982 questionnaires had been returned, indicating a 60 per cent response rate $(982$ out of 1,600 ) and thus a coverage of approximately 12 per cent of all academic staff. The survey responses (both open comments and tickboxes) were entered into a Filemaker Pro database, and numerical data exported into MS Excel, and analysed using Statview. The open comments from the questionnaire were listed according to thematic content and in priority order based upon the number of similar responses.

Semi-structured face-to-face interviews were carried out with senior managers of all twenty-one Scottish HEIs ('institutional visits'); members of staff / educational development units (or equivalent) at nineteen Scottish HEIs; and staff in CAL/LT support units (or equivalent) at seven Scottish HEIs. Interviewees were informed that their answers were confidential and non-attributable. The issues discussed during the interviews included the potential of learning technology, the HEI's current infrastructure and recent developments, planning processes for C\&IT, interactions with LTDI plus value and quality of service, future of funding in this area by SHEFC and preferred funding mechanisms. Detailed notes following the flow of discussion were made at the time, logged later according to the question areas in the interview pro-forma, and subsequently re-analysed in the light of emerging themes.

The SHEFC Teaching Quality Assessment Reports for 1992-6 were analysed for references to learning technology and to IT infrastructure, with comments being scored on a scale from highly complimentary to highly critical.

\section{Results}

\section{Robustness of the survey}

Given the particular importance of determining the validity of survey data, a question always arises as to whether the population sampled is relevant and whether the returns are representative. We believe, for the following reasons, that the survey responses are reasonably representative of the academic population, albeit with some possible bias towards those with an interest and involvement in learning technology: 
- The numbers of returns from each HEI is a reasonable match with its overall size. Thirty per cent of the responses were received from post-1992 institutions and 70 per cent from pre-1992 institutions, which contain 33 per cent and 67 per cent of the teaching staff respectively (SHEFC, 1996).

- Fifty-eight per cent of respondents had not heard of LTDI, 43 per cent had heard of it and half of these (21 per cent of all respondents, i.e. 210 people) had had direct contact with LTDI. Extrapolated to the whole academic population (c. 8,000) these 210 respondents would equate to about 1,800 staff, which accords reasonably well with LTDI's data on attendees and presenters at workshops in the first two years of its operation (c.1,500).

- The distribution of years of experience in higher education of respondents ( 34 per cent up to 10 years, 25 per cent from 10 to 20 years, 31 per cent from 20 to 30 years and 10 per cent over 30 years) indicates that the survey took account of the views of the whole range of higher education experience and did not unduly favour one group. Respondents reported the subject which they taught and these were arranged into four groups - arts, social sciences, sciences and clinical/paraclinical subjects (A, SS, S and $\mathrm{CP}$ respectively). There was a small but significant difference in the mean higher education experience of the respondents - both SS and CP had means of approximately fouteeen years in higher education whereas $A$ and $S$ had means of approximately seventeen years. Many clinical staff begin teaching in higher education later and move in and out more regularly than do other academic staff which would help to explain this difference. For staff in SS likely reasons are less clear although professional background (e.g. commerce) might be part of the explanation.

- It was clearly possible that those staff who are interested or involved in the use of learning technology would preferentially. return forms over their less-involved colleagues. We therefore asked respondents to rate themselves in relation to their peers on experience in use of technology in their teaching (Figure 1). Whilst the distribution is skewed towards those who assessed themselves as having greater experience, there were also comments from some heads of departments that they had found it difficult to identify staff with little or no interest in learning technology, especially where there had been a departmental effort towards its greater use, and thus staff in these departments might rate themselves as higher than average, not recognizing that the average had significantly changed.

The problem of errors in self-evaluation of skills and competencies is well-known in educational research (Moser and Kalton, 1985; Kline, 1993). Respondents may overrate or underrate their skills depending upon the situation. In the absence of additional data, it is not possible to be sure which, if any, bias such errors might create in this case. As we are not concerned here with absolute ratings but with internal comparisons of one self-rated group with another within the same study, the effects of any bias are minimized.

\section{Staff attitudes towards learning technology}

The first section of the questionnaire addressed the topics of the value of learning technology and the extent to which staff were aware of relevant agencies and initiatives. As noted earlier, our respondents were proportionally distributed across the sector, came from a wide range of experience in higher education and rated themselves generally a little above 


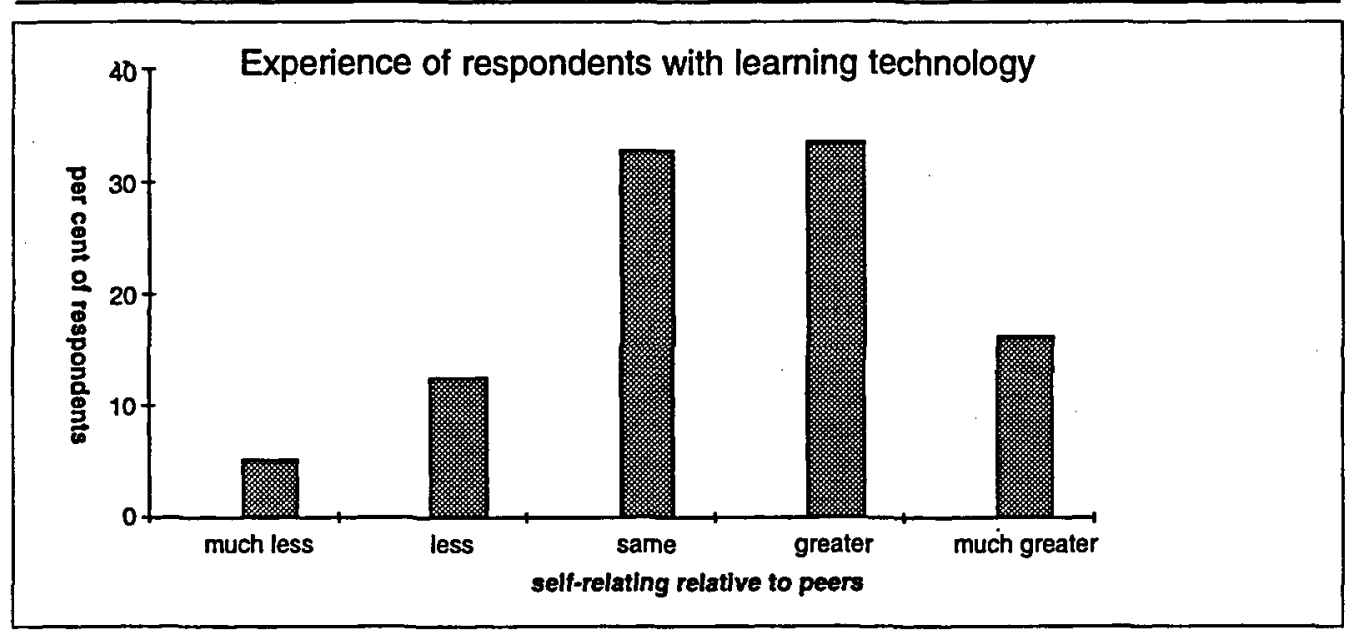

Figure 1: Distribution by self-rated experience with learning technology of academic stoff who responded to the questionnaire. In total 969 staff responded.

average in their experience of use of learning technology. We asked respondents to rate the importance of C\&IT in teaching in their own subject (Table 1). A substantial majority (67 per cent) rated its potential as high ( 24 per cent) or very high ( 43 per cent), and only small numbers ( 3.3 per cent) thought that its potential was low ( 3 per cent) or very low ( 0.3 per cent). Although as expected, most of those who professed higher or much higher than average experience of learning technology rated its potential highly, it is interesting that most of those who professed less or much less experience thought that its potential was moderate or better. One possible bias here may be that, independent of personal experience, returns came disproportionately from staff who feel that this is an important issue. However, as our sample is from around 12 per cent of the entire population, and only a tiny percentage thought that there was little or no value in learning technology, this implies a substantial acceptance across the higher education sector of the opportunities which it offers. This relatively positive picture is backed up for Scotland by data from the CTISS which shows proportionally twice as many contacts with CTI Centres by staff in Scottish HEIs as compared with the rest of the UK (data supplied by CTISS).

Views on the potential of learning technology in higher education were significantly and negatively correlated with years of experience in higher education. Those who thought that the potential was very low or low had an average of nineteen years in higher education, those who viewed the potential as moderate had seventeen years, and those who thought that potential was high or very high had had least experience, sixteen and fourteen years respectively. Some caution must be applied to analysis of these data, however, as there were only three and twenty-nine respondents in the very low and low potential groups respectively. In addition, none of these staff can be viewed as 'junior' since fourteen years in higher education is generally associated with middle-grade staff.

In the face-to-face interviews we were able to explore issues relating to the different views which exist of the potential of learning technology, particularly with respect to efficiency gains versus a role in quality enhancement and/or maintenance. The latter view 


\begin{tabular}{|c|c|c|c|c|c|}
\hline \multirow[b]{2}{*}{$\begin{array}{l}\text { Experience with } \\
\text { learning technology } \\
\text { as compared to } \\
\text { colleagues }\end{array}$} & \multicolumn{5}{|c|}{ Potential for use of learning technology in respondent's subject } \\
\hline & $\begin{array}{c}\text { Very low } \\
\%\end{array}$ & $\begin{array}{c}\text { Low } \\
\%\end{array}$ & $\begin{array}{c}\text { Moderate } \\
\%\end{array}$ & $\begin{array}{c}\text { High } \\
\%\end{array}$ & $\begin{array}{c}\text { Very high } \\
\%\end{array}$ \\
\hline Much less (48) & 2.1 & 10.4 & 41.7 & 39.6 & 6.3 \\
\hline Less (119) & 0.8 & 8.4 & 40.3 & 42.9 & 7.6 \\
\hline Same (314) & 0.3 & 1.9 & 34.4 & 44.6 & 18.8 \\
\hline Greater (321) & 0.0 & 2.2 & 25.5 & 47.0 & 25.2 \\
\hline Much greater (157) & 0.0 & 0.6 & 16.6 & 30.6 & 52.2 \\
\hline
\end{tabular}

Table 1: Respondents' ratings of the potential of learning technology in their subject area versus their self-rating of their experience with the use of learning technology in comparison to their colleagues. Total numbers of respondents in each self-rating category are shown in parentheses in Column 1. The bold entries highlight the small numbers of staff with little experience of leaming technology who felt that leaming technology was of low or very low potential in teaching of their subject.

predominated, albeit tempered by hopes that in some areas, especially assessment and student support, there could be savings in staff time. Few senior staff who were interviewed saw opportunities for overall savings in the short or medium term, as the cost in staff time to select, implement and evaluate the use of learning technology is high and the costs in rolling upgrades of infrastructure are ever increasing. This view was also supported by comments from learning technology support staff. No one voiced antipathy to the view that there are opportunities for quality enhancement, although some clearly felt driven by external forces to adopt a pace which might not be that of their own choosing. Overall, the task of convincing the majority of the Scottish higher education sector of the opportunities offered by learning technology appears to be well in hand. Converting this positive perception into action, however, is an entirely different matter.

It is important to note here that the view from respondents to the last section of our questionnaire (those who gave written comments and thus had strong views) was that many academic staff were hostile to learning technology or unwilling to use it. However, this contrasts strongly with the general opinion expressed in the first section of the questionnaire, namely that, irrespective of whether they had heard of LTDI or other initiatives or of their experience, most staff thought that C\&IT had moderate or greater potential in higher education. Whilst the failure of many to convert that view into action may be interpreted by committed staff as antipathy or reluctance, it may actually have more to do with how competing demands are prioritized or with practical difficulties encountered. Thus, relying on strongly expressed views from highly committed staff may in fact lead to an inappropriately negative impression of the state of the sector overall and its attitudes towards learning technology.

\section{Awareness of 'initiatives' and learning technology agencies}

Given the generally positive view of learning technology taken by the questionnaire respondents, it is not surprising that their awareness of the agencies associated with its promotion is high (Table 2). Only 27 per cent of respondents (261/969) had not heard of any of the agencies, whilst only 45 per cent (429/969) reported not having had any direct 


\begin{tabular}{l|rrrrrr|rrrrrr}
\hline $\begin{array}{l}\text { Learning technology } \\
\text { experience with } \\
\text { respect to } \\
\text { colleagues }\end{array}$ & \multicolumn{4}{|c|}{ Heard of agency (\%) } & \multicolumn{5}{c}{ Contact with agency (\%) } \\
\hline Much less (49) & 24.5 & 20.4 & 20.4 & 12.2 & $\mathbf{4 0 . 8}$ & 6.1 & 0.0 & 6.1 & 4.1 & 0.0 & $\mathbf{8 . 2}$ \\
Less (120) & 43.3 & 30.0 & 25.8 & 15.8 & $\mathbf{6 0 . 8}$ & 7.5 & 5.8 & 11.7 & 5.8 & 2.5 & $\mathbf{2 6 . 7}$ \\
Same (317) & 48.6 & 32.5 & 34.1 & 18.9 & $\mathbf{6 4 . 0}$ & 12.6 & 9.1 & 11.4 & 8.8 & 0.9 & $\mathbf{3 5 . 3}$ \\
More (325) & 69.2 & 100.0 & 56.3 & 25.8 & $\mathbf{8 2 . 5}$ & 19.1 & 26.8 & 25.8 & 22.2 & 3.7 & $\mathbf{5 5 . 4}$ \\
Much more (158) & 78.5 & 100.0 & 73.4 & 38.6 & $\mathbf{9 1 . 1}$ & 32.3 & 39.2 & 35.4 & 39.9 & 7.6 & $\mathbf{6 9 . 6}$ \\
Total (969) & 58.5 & 65.2 & 46.2 & 23.7 & $\mathbf{7 3 . 1}$ & 17.0 & 19.1 & 19.9 & 17.8 & 3.1 & $\mathbf{4 5 . 2}$ \\
\hline
\end{tabular}

Table 2: Awareness of, and contact with, learning technology ogencies by academic staff grouped by self-rating of their own experience with the use of learning technology relative to that of their colleagues. The numbers of respondents. in each experience group is shown in parentheses. Other columns show the numbers of respondents in each experience category as percentages of total respondents in that category. The sixth and twelfth columns (in bold) show owareness or contact with any one of the four agencies. Only 969 out of the 982 respondents could be included in this analysis due to incomplete returns. Very few respondents reported contact with all agencies and so these data are omitted.

contact. As might be expected, both these types of respondents were clustered mostly amongst those who rated themselves as less or much less experienced than their peers with learning technology. Of those respondents who had heard of these agencies, slightly more had heard of LTDI than of CTI (65 per cent vs. 58 per cent) and almost the same percentage had had contact with each (19 per cent and 20 per cent respectively), showing that LTDI and CTI had made direct contact with almost a third of the staff who had heard of them. TLTP was slightly less well-known, followed by ITTI. The low knowledge of ITTI is not surprising given that the post-1992 HEIs were too late to get the full benefit from it and its range of actions and targets was much more restricted than other agencies. What is impressive is that 73 per cent of all respondents in Scottish HEIs had heard of one of these agencies and 45 per cent had had some contact, mainly through workshops and seminars.

Influence on awareness of experience with learning technology

Not unexpectedly, more learning technology-experienced staff had greater awareness of, and contact with, relevant agencies than had their less experienced colleagues. Awareness reached 100 per cent for LTDI and 91 per cent of the most experienced group had heard of at least one of the four agencies. It is striking that 41 per cent of the least experienced staff had also heard of one of the four agencies and 55 per cent (93/169) of the combined 'much less' and 'less' experienced groups had heard of one agency. Even allowing for the fact that there is some skew in the distribution of self-rating in favour of the more experienced groups, and that survey forms may have been selectively returned by those with above average interest in learning technology, these percentages imply a substantial impact by these agencies on the consciousness of academic staff in Scotland. The key role of subject focus in use of C\&IT in learning and teaching is demonstrated by the relatively high awareness of the CTI Centres amongst the less experienced groups. 
Influence on awareness of length of time in higher education

Awareness of, or contact with, agencies was not influenced by the number of years in higher education, contradicting stereotypes of 'older' staff being less interested in new teaching methods or 'younger' staff being too busy with research. However, more recently appointed staff did appear to be more involved in learning technology. Staff who rated themselves 'much less' or 'less experienced' with C\&IT than their colleagues had an average of almost nineteen years in higher education, whereas those who self-rated as of the 'same', 'greater' or 'much greater' experience than their peers all had an average of approximately fifteen years in higher education. These data compare favourably with those relating staffs' attitudes to learning technology and the length of time they had spent in higher education.

Influence on awareness of subject area

Contrary to the prevailing stereotype, there was no significant difference between any of the broad subject areas of arts (A), clinical/paraclinical (CP), sciences (S) and social sciences (SS) with respect to respondents' views of the potential of learning technology in their area. There was also no difference in the self-ratings of learning technology experience between staff in A, SS and S but, in contrast, respondents in clinical/ paraclinical subjects gave consistently lower self-ratings than the other areas. Their lower self-rating correlates with significantly lower awareness of agencies set up to promote and support use of C\&IT in education. Twice as many clinical/paraclinical respondents. (43 per cent) had not heard of any of the four agencies (LTDI, CTI, ITTI, TLTP) in comparisons with S and SS ( 22 per cent and 25 per cent respectively), with arts slightly higher at 29 per cent. Similar results were found when contact with agencies was analysed. This probably partly explains our observation that significantly more staff in large institutions had heard of none of the agencies, as three of the large Scottish HEIs have medical schools, and the lower number of years of experience of staff in higher education in these subjects.

Influence on awareness of staff in different types of higher education institution

Staff in smaller institutions ( $<200$ academic staff) were more aware of the learning technology agencies, and more of them had heard of all four agencies, than their colleagues in medium (200-500 staff) and larger institutions ( $>500$ staff), perhaps reflecting the ease with which information can be disseminated around them (data not shown). It is also the case that in this population in Scotland there are several monotechnic institutions (teacher training colleges, art colleges) where the range of subjects taught is quite small. At the opposite end of the awareness spectrum, significantly more staff in large institutions had not heard of any of the agencies, and the presence in three of these of large clinical and paraclinical subjects is likely to be part of the explanation. There was no significant. difference between staff as a whole in pre- and post-1992 institutions in awareness of, or contact with, any of the four agencies.

\section{Barriers to use of learning technology in teaching and learning}

In spite of the broadly positive views of the value of learning technology, it should be noted that we observed, and respondents registered, a strong feeling that many serious barriers still exist which inhibit its widespread use by staff in their teaching. A similar set of themes reverberated throughout the open comments made in the questionnaire, whether they referred to reasons for not considering it feasible to make use of C\&IT in learning and teaching, the obstacles encountered in trying to develop such activities, or the challenges identified as faced by agencies. Staff with greater experience of learning technology were 
ALT-J Volume 8 Number 2

\begin{tabular}{|c|c|c|c|}
\hline $\mathrm{Q} 8$ & $Q 10+11$ & Q15a & Q15d \\
\hline $\begin{array}{l}\text { Reasons for not } \\
\text { considering use of } \\
\text { learning technology in } \\
\text { teaching }\end{array}$ & $\begin{array}{l}\text { Factors inhibiting } \\
\text { Jearning technology } \\
\text { development }\end{array}$ & $\begin{array}{l}\text { Challenges faced by } \\
\text { LTDI }\end{array}$ & Ways forward \\
\hline $\begin{array}{l}\text { insufficient resources, } \\
\text { time, \& priority }(50 \%)\end{array}$ & $\begin{array}{l}\text { lack of suitable software } \\
(40 \%)\end{array}$ & $\begin{array}{l}\text { resistance to use of } \\
\text { technology generally } \\
\text { amongst teaching staff } \\
(33 \%)\end{array}$ & $\begin{array}{l}\text { institutional/subject } \\
\text { area/departmental } \\
\text { support (39\%) }\end{array}$ \\
\hline lack of $\pi$ skills $(25 \%)$ & lack of staff time (34\%) & lack of staff time $(15 \%)$ & $\begin{array}{l}\text { support for software } \\
\text { development }(25 \%)\end{array}$ \\
\hline \multirow[t]{6}{*}{$\begin{array}{l}\text { course characteristics } \\
\text { not favourable }(25 \%)\end{array}$} & $\begin{array}{l}\text { insufficient local } \\
\text { institutional support } \\
(23 \%)\end{array}$ & $\begin{array}{l}\text { relative status of } \\
\text { teaching priorities \& } \\
\text { incentives (13\%) }\end{array}$ & $\begin{array}{l}\text { support for other } \\
\text { initiatives }(11 \%)\end{array}$ \\
\hline & $\begin{array}{l}\text { general lack of resources } \\
(11 \%)\end{array}$ & $\begin{array}{l}\text { developing a } \\
\text { recognized functional } \\
\text { niche }(11 \%)\end{array}$ & $\begin{array}{l}\text { reduce staff } \\
\text { workloads (1 1\%) }\end{array}$ \\
\hline & $\begin{array}{l}\text { lack of appropriate } \\
\text { hardware }(9 \%)\end{array}$ & $\begin{array}{l}\text { lack of institutional } \\
\text { resources }(9 \%)\end{array}$ & staff $I T$ training $(7 \%)$ \\
\hline & & $\begin{array}{l}\text { lack of staff IT skills } \\
(9 \%)\end{array}$ & hardware $(7 \%)$ \\
\hline & & $\begin{array}{l}\text { lack of appropriate } \\
\text { hardware }(7 \%)\end{array}$ & \\
\hline & & $\begin{array}{l}\text { lack of appropriate } \\
\text { software }(4 \%)\end{array}$ & \\
\hline
\end{tabular}

Table 3: Themes present in open-ended responses to selected questions in the academic staff questionnaire. Values in parentheses are the percentoges of comments which contained each theme.

more inclined to supply these comments than were less experienced staff; 12.5 per cent of all respondees gave comments; 8 per cent of the much less experienced group did so as compared with 17 per cent of the much more experienced group. As the less experienced groups were smaller than the more experienced group, the majority of the open comments came from 'experts' rather than from 'novices'.

The themes which emerged in the open comments questions of the paper survey are summarized in Table 3.

These difficulties include:

- lack of time, once high priority tasks have been accounted for, which is the principal limitation for many staff, particularly those in the former SOED HEIs; 
- perceptions of the relatively low status, and hence rewards, accorded to teaching compared with research, especially given the pressures of the Research Assessment Exercise with its significant implications for funding;

- lack of reliable and adequate infrastructure, including technical support to deliver courseware at reasonable cost in terms of academic effort;

- lack of appropriate courseware in some subjects;

- lack of basic C\&IT skills, especially in HEIs where the IT infrastructure is quite new.

The difficulties enumerated by staff are, of course, not all unique to the use of learning technology. Some apply to all teaching innovations, but perhaps they become more obvious where C\&IT is concerned due to the acute way in which it forces these barriers into the open.

However, whilst improvements in IT infrastructure are relatively easily made, releasing time for staff to be creative in their teaching, and rewarding and valuing this alongside other activities will be less easy to achieve. Comments in the questionnaire stressed the importance of incentives - 'convincing universities that teaching skills are important' and 'convincing lecturers that it is in their career interests to invest time and effort' in 'what are [the] time-intensive activities' entailed in course development. Where the topic arose within institutional visits, many HEIs admitted that progress in this regard was still slow, or perceived to be so by many staff. This important issue clearly affects innovation in teaching generally, and is not restricted to the use of learning technology.

Finally, culture change is slow. The cyclic nature of academic years results in changes to courses often lagging one or two years behind the decision to change, partly because of the need to plan ahead but also because changes in course structures, particularly where they impinge on assessment procedures, need to be reviewed and approved by relevant committees within the institutions. Thus many effects of learning technology agencies may not be seen for another one or two years. This slow change is not necessarily a negative phenomenon. For change to be embedded, there has to be movement on a broad front, taking a substantial number of staff along with it rather than a small number of enthusiasts, and for this to happen enough time and focused support will be needed. However, although these changes may be slow, this survey does indicate that the process is firmly underway.

\section{SHEFC Quality Assessment Reports}

The importance of the Teaching Quality Assessment process in shaping course design and delivery cannot be ignored. Despite the present uncertainty about the overall format of TQA in the next few years, ensuring that it reflects the need to identify and comment on use of learning technology would assist those staff who are working to introduce it into their courses.

By the end of the academic year 1995-6, SHEFC had published 224 Quality Assessment Reports covering 32 cognate areas (SHEFC 1992-6). The Quality Assessment Framework mentions IT infrastructure in teaching and learning in Section $\mathrm{E}$, and innovative teaching methods making use of all facilities in Section G. As assessors use this framework to structure their assessment and reports, these peer reviews are an important guide to the 
current level of use of C\&IT and also to the importance attached to this by the assessors (in itself an interesting insight into the minds of respected members of the profession).

A brief analysis of references in the reports to provision and use of C\&IT was made by rating comments on a 'critical' to 'complimentary' scale from - - - through 0 to +++ . This exposed some interesting trends. The first was the relatively high importance placed on provision of computers and software in some subjects (e.g. theology, mathematics and statistics) and the relatively low importance placed on it in others (e.g. civil engineering, social work). Indeed some assessment teams rarely mentioned the topic at all, and there was often inconsistency in reporting within single cognate areas, perhaps reflecting variations in composition of assessment teams. What was generally the case was the paucity of direct reference to the use of technology in teaching and learning as opposed to the provision of a hardware/software infrastructure. By looking for indirect evidence of the absence of use of C\&IT by such comments as 'emphasis on traditional teaching methods', 'little use of innovative teaching methods' etc., it is possible to gauge the lack of use of learning technology. The outcome of this brief analysis showed that many departments across Scotland had yet to make significant innovations in their teaching (indeed to move to a more student-centred view with the emphasis on learning) despite some being rated overall in the reports as 'excellent'.

There are caveats to this analysis. Firstly, the 'Confidential Notes for the Institution' are not publicly available and secondly the lead time for introduction of substantive change to courses is long, and some reports did comment on changes under way. Nevertheless, taken as a whole the reports do suggest the need for continued support to this area.

\section{Conclusions}

These surveys and interviews provide the first in-depth information on the awareness of, and views about, learning technology of a wide range of staff in Scottish higher education. It is clear that the efforts of those involved in awareness-raising have paid off, in that few of our respondents were unaware of any LT agency and many knew of several. This was true even for staff who rated themselves as lower in experience with learning technology than their colleagues. Substantial numbers had also made contact in some way with at least one agency. Very few staff at any level viewed C\&IT as of little or no value to teaching in their subject, most seeing it as being of moderate to significant value, but more for quality enhancement than for efficiency gains. There was no significant difference in these views between broad subject areas and age or type of HEI. These positive attitudes are also reflected in the Dearing Report (DfEE, 1997, 3rd Report) where a survey of academic staff showed that most of the effected changes in teaching were based upon new technologies and were designed to benefit students rather than for institutional or efficiency gains.

The data are also broadly in line with later findings in the UK. In the recent survey of use of TLTP materials in UK higher education (HEFCE, 1999b) 31 per cent of academic staff respondents reported that they thought their subject 'lends itself greatly' to use of C\&IT in learning and teaching, and 60 per cent felt that it 'lends itself moderately'. Of these staff, 95 per cent thought that there was scope for expansion in the use of C\&IT in the teaching of their subject at moderate or substantial levels.

In contrast with this, the most experienced staff and especially those in learning technology 
support roles, had a more pessimistic view and felt that many of their colleagues were hostile or unwilling to take the challenge of adopting it in their teaching. This contrast of perspectives is important given the use of key informants in much of what informs policymaking in higher education.

However, significant barriers to the use of learning technology do still exist, especially the lack of time for academic staff and the relative importance placed on research as compared to teaching. These themes are also evident in other studies of this subject. For instance, in the United States, the Campus Computing 1999 survey of 530 senior computing services staff in colleges nation-wide notes that 'assisting faculty to integrate information technology into instruction' is now the major challenge (Green, 1999). Although the majority of institutions had establisned a centre to support the use of learning technology, very few had formal recognition of such C\&IT-based teaching in their academic staff review and promotion procedures. In the UK, a comparable lack of embedded institutional commitment to new technologies is evident in the findings of the recent study of C\&IT materials in higher education and further education (HEFCE, 1999a) in which the learning and teaching strategies of all English HEIs were analysed. Only a small number of HEIs had identified enhanced use of C\&IT as a major part of their strategy, despite the fact that a wide variety of developments are taking place within all of them. Similar barriers to widespread uptake of learning technology were identified - lack of commitment at senior management levels, research dominance over teaching, and technical and pedagogical support issues. It was as a consequence of concerns expressed in the Dearing Report about the need to give more support to innovation in learning and teaching (DfEE, 1997), that the Institute for Learning and Teaching (ILT) was established to provide a counterweight to the dominance of research in higher education, promote a culture shift and 'a re-think of institutional priorities'.

Finally, there is little evidence that the TQA exercise in Scotland in the period 1992-6 took any systematic stance towards the use of learning technology in the subjects which were assessed, despite its stated involvement in the rationale for the establishment of the QA system. This may give us an important insight into the views of senior members of the professions who formed the peer assessment panels, and perhaps raises concern with respect to their awareness of, and concern for, innovation in the teaching of their subject.

Although the data in this study come from responses from staff who were to a large extent self-selecting in their decision to return surveys about C\&IT in learning and teaching, large numbers of academic staff did return them. Even allowing for some bias, the absolute number of staff in the Scottish higher education sector with a positive view of the value of C\&IT is encouraging and may be taken as an indicator that it has moved from a fringe to a mainstream concern for academic staff in all subject areas. Supporting those staff and building upon that base is an urgent task for both the institutions and the Funding Councils.

\section{Acknowledgements}

We should like to acknowledge the input of all those who helped us in the course of our work, in particular the staff of the Scottish HEIs and agencies who gave generously of their time to participate in interviews, and for the open way in which they expressed their 
opinions. We also thank the nearly 1,000 academic staff who took the time to complete our questionnaire, and particularly those who provided written comments to round out their responses. Analysis of the data from the questionnaire would not have been possible without the efficient processing of the survey forms and letters by the project secretary, Mrs Mary Scott.

\section{References}

Davies, G. (1995), Foreword to TLTSN Case Studies: Some Senior Management Issues, Bristol, Higher Education Funding Council for England.

DfEE (1997), Higher Education in the Learning Society - The Dearing Report, Report of the National Committee of Enquiry into Higher Education, London, Department for Education and Employment, http://www.leeds.acukleducollncihe (last accessed 2 Feb. 2000).

Green, K. C. (1989-99), Campus Computing Survey, Campus Computing, Encino, California, USA, http://www. campuscomputing.net/ (last accessed 2 Feb. 2000).

HEFCE (1999a), Communications and Information Technology Materials for Learning and Teaching and UK Higher and Further Education, Report 99/60 Bristol, Higher Education Funding Council for England.

HEFCE (1999b), Uses of TLTP Materials in UK Higher Education, Report 99/39 Bristol, Higher Education Funding Council for England.

ITATL (1997), Information Technology Assisted Teaching and Learning in Higher Education, Bristol, Higher Education Funding Council for England.

Kline, P. (1993), Handbook of Psychological Testing, London, Routledge.

Moser, C. A. and Kalton, G. (1985), Survey Methods in Social Investigation, Aldershot, Gower.

SHEFC (1992), Circular 8/92, Edinburgh, Scottish Higher Education Funding Council.

SHEFC (1992-6), Teaching Quality Assessment Reports, Edinburgh, Scottish Higher Education Funding Council.

SHEFC (1996), Statistical Bulletin 3/96, Edinburgh, Scottish Higher Education Funding Council. 